\title{
PRECISÃO EXPERIMENTAL E TAMANHO DA ÁREA DE EXPERIMENTOS DE CAMPO COM FRUTEIRAS E OUTRAS PLANTAS PERENES ARBÓREAS EM FUNÇÃO DA UNIDADE EXPERIMENTAL E DO NÚMERO DE REPETIÇÕES ${ }^{1}$
}

\author{
ADROALDO GUIMARÃES ROSSETTI²
}

\begin{abstract}
RESUMO - Um problema dos experimentos de campo com plantas perenes e frutíferas arbóreas é o tamanho da área, devido ao porte das plantas que normalmente exigem largos espaçamentos. É muito freqüente, nesses experimentos, o uso de parcelas grandes, em detrimento do número de repetições, com a justificativa de diminuir a área experimental, a mão-de-obra e o conseqüente custo da pesquisa. Essa prática, contudo, traz prejuízos à precisão das estimativas dos parâmetros e à aplicação eficiente de testes estatísticos. Este trabalho foi realizado com o objetivo de mostrar que o aumento do número de repetições com o uso de parcelas pequenas aumenta a precisão dos experimentos, das estimativas do erro experimental e dos efeitos de tratamentos, favorece a detecção de diferenças significativas entre os tratamentos e contribui para diminuir a área experimental. Desenvolveu-se um procedimento que associa o tamanho da unidade experimental ao número de repetições, pela minimização da variância da média de cada tratamento, que permite o uso de maior número de repetições, para aumentar a precisão dos testes, obter maior uniformidade no experimento e melhorar a qualidade das pesquisas. Os resultados obtidos permitiram observar que o uso de parcelas pequenas favorece o aumento do número de repetições, permite obter melhores estimativas do erro experimental, dos efeitos de tratamentos e dos parâmetros, além de dar mais eficiência aos testes estatísticos a serem aplicados aos dados. Observou-se, também, diminuição substancial do número de plantas necessárias aos experimentos e do tamanho da área experimental.
\end{abstract}

Termos para indexação: variância da média de cada tratamento, área experimental, coeficiente de correlação intraclasse, bordadura.

\section{PRECISION AND AREA SIZE IN FIELD TRIALS WITH PERENNIAL PLANTS AND FRUITS TREES AS FUNCTION OF THE PLOT AND NUMBER OF REPLICATES}

\begin{abstract}
Area size is a matter of concern in field trials with perennial plants and fruits trees, as they usually require large spacings. Big plots and small number of replications are frequently used in these cases to justify the reduction in the experimental area, labor and consequently in the costs of the research. However, this procedure impairs the precision of parameter estimations and the efficiency in application of statistical tests. This work has been carried out with the aim of demonstrating that precision of trials and estimations of experimental error and effects of treatments cam be improved, a detection of significant differences among treatments are favored, and the experimental area is reduced by increasing the number of replicates and using small plots.

A procedure has been developed that associates size of plot and number of replicates by the mean variance of each treatment, allowing the employment of a larger number of replicates to increase test precision, increase trial uniformity and improve quality of research. The results showed that the use of small plots favors the increase in number of replicates leads to the obtention of better estimations of experimental error effects of treatments and parameters besides allowing more efficiency to statistical tests used. A substantial reduction in the number of plants needed for the trial as well as in the size of experimental area was also observed.
\end{abstract}

Index terms: Experimental area, treatment mean variance, and intraclass correlation coefficient guard rows.

\section{INTRODUÇÃO}

Os experimentos de campo com fruteiras e outras plantas perenes arbóreas ocupam, em geral, grandes áreas, pois exigem largos espaçamentos, trazendo, segundo Rossetti (1994), alguns problemas, com reflexos nos resultados das pesquisas. Usam-se experimentos com parcelas grandes e poucas ou sem repetições, para obter maior "stand" e facilitar o manejo. Pergunta-se, também, qual o número mínimo de repetições e o tamanho ideal da parcela que devem ter os tratamentos desses experimentos, para se ter bons resultados. A repetição visa a estimar melhor o erro experimental, tão importante aos testes dos efeitos de tratamentos e outros parâmetros dos modelos, permite distribuir os tratamentos em maior espaço do ambiente, validando a extrapolação dos resultados. Vários autores, como Dagnelie (1975), demonstram que quanto maior for o número de repetições, melhor será a estimativa do erro experimental e dos efeitos de tratamentos. Ocorre que, dependendo do número de tratamentos, do delineamento destes, do tamanho da parcela e do delineamento experimental utilizados, o experimento pode crescer tanto e tornarse impraticável. Por isso, usam-se parcelas com grande número de plantas, em detrimento do número de repetições, o que é

\footnotetext{
1 (Trabalho 047/2001). Recebido: 19/02/2001. Aceito para publicação: 10/07/2001.

2 Pesquisador, Embrapa Agroindústria Tropical (CNPAT), Rua Dra. Sara Mesquita, 2270 - Pici. Caixa Postal 3761, CEP 60511-110 Fortaleza CE. adroaldo@cnpat.embrapa.br
} 
grave, pois parcelas grandes têm maior variância, segundo demonstraram Rossetti et al. (1996), aumentando a variância da média de cada tratamento. Rossetti \& Pimentel Gomes (1987) recomendam o uso de parcelas de tamanho ótimo, que podem ser estimadas de dados de experimentos com a cultura de interesse, em condições semelhantes às da pesquisa proposta.

A determinação do número ideal de repetições é um problema que tem sido bastante estudado e muitas soluções têm sido propostas, mas, segundo Pimentel Gomes (1990), nenhuma é inteiramente satisfatória. O uso de parcelas de tamanho ótimo, segundo Rossetti \& Pimentel Gomes (1983), associado a delineamentos ou técnicas experimentais apropriados, contribui para reduzir a variabilidade, normalmente existente nesses cultivos. Isso requer tratamentos homogêneos, com mínima variância, dentro da parcela. Nos materiais propagados sexuadamente, é ainda muito maior a necessidade de cuidados tanto do binômio tamanho da parcela e número de repetições como de outras técnicas experimentais para obter bons resultados. Os experimentos com grande número de tratamentos, independentemente da área de pesquisa que normalmente requerem grandes áreas, têm particular importância nesse contexto.

Este trabalho foi realizado com o objetivo de mostrar que o uso de parcelas pequenas, nos experimentos com fruteiras e outras plantas perenes arbóreas, favorece o aumento do número de repetições, melhora as estimativas do erro experimental, dos efeitos dos tratamentos e outros parâmetros do modelo, diminuindo a área do experimento e o número de plantas necessárias.

\section{MATERIAL E MÉTODOS}

Considere-se um experimento com $\mathbf{t}$ tratamentos, em $\mathbf{b}$ blocos casualizados, com $\mathbf{k}$ plantas úteis por parcela e dados obtidos de cada planta, cujo modelo estatístico, segundo Pimentel Gomes (1984), é: $Y_{\mathrm{ijk}}=\mathrm{m}+\mathrm{t}_{\mathrm{i}}+\mathrm{b}_{\mathrm{j}}+\mathrm{e}_{\mathrm{ij \textrm {k }}}, \mathrm{l}$, onde: $\mathbf{m}$ é a média, $\mathrm{t}_{\mathrm{i}}(\mathrm{i}=1,2$, ..., t) é o efeito do i-ésimo tratamento, $b_{i}(j=1,2, \ldots, b)$ é o efeito do j-ésimo bloco, $\mathbf{e}_{\mathrm{ijk}}$ são aleatórios, com $\mathrm{E}\left(\mathrm{e}^{2}{ }_{\mathrm{ijk}}\right)=\sigma^{2}, \mathrm{E}\left(\mathrm{e}_{\mathrm{ijk}_{\mathrm{j}}} \mathrm{e}_{\mathrm{i}^{\prime} j^{\prime} \mathrm{k}}\right)=0$ para $(i, j)^{11}\left(i^{\prime}, j j^{\prime}\right), E\left(e_{i j k} e_{i i^{\prime} k^{\prime}}\right)=\rho \sigma^{2}$, onde $\rho$ é o coeficiente de correlação intraclasse (a correlação que deve existir entre parcelas vizinhas) e $\sigma^{2}$ é a variância experimental, cuja análise de variância é a da Tabela 1:

TABELA 1 - Análise de variância dos componentes do modelo (1), do delineamento experimental.

\begin{tabular}{lccc}
\hline Fonte de variação & GL & QM & E(QM) \\
\hline Blocos & $(\mathrm{b}-1)$ & - & - \\
Tratamentos & $(\mathrm{t}-1)$ & - & - \\
Resíduo (a) & $(\mathrm{b}-1)(\mathrm{t}-1)$ & $\mathrm{V}_{1}$ & $\sigma^{2}[1+(\mathrm{k}-1) \rho]$ \\
Resíduo (b) & $\mathrm{b} \mathrm{t}(\mathrm{k}-1)$ & $\mathrm{V}_{2}$ & $\sigma^{2}(1-\rho)$ \\
\hline
\end{tabular}

Na Tabela $1, V_{1}$ é a estimativa da variância relativa às parcelas, $V_{2}$ a referente a plantas dentro de parcelas e $\mathbf{k}$ é o número de plantas úteis da parcela.
Com $\rho \geq 0, E\left(V_{1}\right)>E\left(V_{2}\right)$, para $\mathrm{k}>1$. Mas com $\rho<0$ tem-se a possibilidade de obter $\mathrm{E}\left(\mathrm{V}_{1}\right)<\mathrm{E}\left(\mathrm{V}_{2}\right)$, o que é importante, pois devese ter sempre $\sigma^{2}[1+(k-1) \rho] \geq 0$, ou seja: $\rho>\frac{1}{k-1}$, para $(\mathrm{k}>1)$.

Essa expressão indica que, aumentando o valor de $\mathbf{k}$, o valor de $\rho \mathbf{r}$, se inicialmente negativo, estará cada vez mais próximo de zero, isto é, para $\rho<0$, tem-se: $\lim \rho=0 \forall \mathrm{k} \rightarrow \infty$.

Em qualquer caso, tem-se sempre, $-\frac{1}{k-1} \leq \rho \leq 1,(\mathrm{k}>1)$.

Da análise de variância da Tabela 1, obtém-se um estimador de $\rho$, pela expressão:

$\hat{\rho}=\frac{\mathrm{V}_{1}-\mathrm{V}_{2}}{\mathrm{~V}_{1}+(\mathrm{k}-1) \mathrm{V}_{2}},(\mathrm{k}>1)$, concluindo-se que: $\mathrm{V}_{1} \geq \mathrm{V}_{2} \Rightarrow \hat{\rho}>0$

e $\mathrm{V}_{1}<\mathrm{V}_{2} \Rightarrow \hat{\rho}<0$.

De outro modo, sendo $V_{1}$ e $V_{2}$ ambos positivos, tem-se necessariamente: $-\frac{1}{k-1}<\hat{\rho}<1, \quad \forall \quad \mathrm{k}>1$.

Note-se que, para $0<\rho<1$, tem-se: $\frac{\rho}{1-\rho}=\left(\frac{\sigma_{1}}{\sigma_{2}}\right)^{2}$

A estimativa do coeficiente de variação será obtida pela variância relativa de cada parcela cuja expressão é:

$\mathrm{V}_{1}=\sigma^{2}[1+(\mathrm{k}-1) \hat{\rho}]$.

Conclui-se, portanto, que, sendo $\mathbf{m}$ a estimativa da média geral, o coeficiente de variação, relativo ao resíduo (a), referente a parcelas, é obtido pela expressão:

$\mathrm{CV}=(100 / \mathrm{km}) \sqrt{\sigma^{2}[1+(k-1) \hat{\rho}]}, \mathrm{CV}=(100 \sigma / \mathrm{m}) \sqrt{\frac{1-\hat{\rho}}{k^{2}}+\frac{\hat{\rho}}{k}}$,
$\mathrm{CV}=(100 \sigma / \mathrm{m}) \sqrt{\frac{(1-\hat{\rho})+k \hat{\rho}}{k^{2}}}, \mathrm{~K}>0, \hat{\rho} \geq 0$.

A média de plantas $\mathbf{m}$ é uma função decrescente de $\mathbf{k}$.

Com $\hat{\rho} \geq 0$, o máximo do $C V$ se dá para $\mathrm{k}=1$, pois o radicando

é função decrescente de $\mathbf{k}$. Se $\hat{\rho}=1$, o primeiro termo é nulo, mas isso não altera o resultado.

Com $\hat{\rho}<0$, o numerador do radicando $[(1-\hat{\rho})+\mathrm{k} \hat{\rho}]$ decresce quando $\mathbf{k}$ cresce, mas seu valor é restringido pela condição necessária de que se tenha $[(1-\hat{\rho})+\mathrm{k} \hat{\rho}] \geq 0$. Ao mesmo tempo, cresce o denominador $\mathbf{k}^{2}$, de modo que o radicando é monotonicamente decrescente, para $\mathrm{k} \geq 1$. Isso indica que o coeficiente de variação é função decrescente de $\mathbf{k}$, o que levaria ao uso de parcelas grandes, contribuindo para o aumento da variância da média de cada tratamento, dificultando a detecção de diferenças significativas entre eles, o que realmente se deseja. Para isso, é necessário reduzir a variância dentro da parcela, o que implica reduzir a variância da média de cada tratamento, sem aumentar o número de plantas da parcela, isto é, para uma área fixa ou um número fixo de plantas, tornar mínima a variância da média de cada tratamento a fim de que se possam detectar diferenças significativas entre eles, quando estas existirem.

Considerando o modelo expresso em (1), a variância da média de $\mathbf{r}$ repetições de cada tratamento é obtida pela expressão: 


$$
\mathrm{V}(\hat{m})=\left(\frac{\sigma^{2}}{k r}\right)[1+(\mathrm{k}-1) \hat{\rho}] \text {, para } \mathrm{k}, \mathrm{r}>1 \text { e } \hat{\rho}>0 \text {. }
$$

Não havendo bordadura entre as parcelas e sendo $\mathbf{N}$ o número de plantas por tratamento, tem-se $\mathrm{N}=\mathrm{kr}, \log$ :

$\mathrm{V}(\hat{m})=\left(\frac{\sigma^{2}}{N}\right)[1+(\mathrm{k}-1) \hat{\rho}]$.Com $\hat{\rho}>0$, essa variância é mínima para $\mathrm{k}=1$, isto é, para parcelas formadas de uma única planta.

Para $\hat{\rho}<0$, essa variância é função decrescente de $\mathbf{k}$, ou seja:

$\mathrm{f}(\mathrm{k})=\mathrm{V}(\hat{m})=\left(\frac{\sigma^{2}}{N}\right)[1+(\mathrm{k}-1) \hat{\rho}]$.

Havendo bordadura $(b=1 / 2, b=1, b=2)$, conforme se usar meia bordadura, bordadura completa ou simples, ou bordadura dupla entre as parcelas, de $\mathbf{k}$ plantas úteis, em $\mathbf{n}$ fileiras, sendo $\mathbf{N}$ o número total de plantas por tratamento, $\mathrm{N}=\mathrm{Kr}$ ( $\mathrm{N}$ constante) $\mathrm{e}$ r o número de repetições, $o$

$K=(n+2 b)\left(\frac{k}{n}+2 b\right)$ ou $K=\left(1+\frac{2 b}{n}\right)(k+2 b n), n>1$

Como $\mathbf{N}$ é o número total de plantas por tratamento e $\mathbf{r}$ o número de repetições: $\mathrm{N}=\mathrm{Kr}=\left(1+\frac{2 b}{n}\right)(k+2 n b) r$, e a variância da média do tratamento será então:

$$
\mathrm{V}(\hat{m})=\frac{\sigma^{2}}{K r}=\left(1+\frac{2 b}{n}\right)(k+2 b n) r \Rightarrow \mathrm{V}(\hat{m})=\frac{\sigma^{2}}{N}\left(1+\frac{2 b}{n}\right)\left(1+\frac{2 b n}{k}\right)[1+(k-1) \hat{\rho}], \mathrm{k}>1 \mathrm{e}
$$$$
\hat{\rho}>0 \text {. (3) }
$$

$\mathrm{O}$ mínimo dessa função, para $0 \hat{p}<1$, será obtido igualando-se a zero as derivadas parciais $\partial \mathrm{V} / \mathrm{dk}$ e $\partial \mathrm{V} / \mathrm{dn}$, respectivamente, para o número $\mathbf{k}$, de plantas úteis e o número $\mathbf{n}$, de fileiras, da área útil da parcela. Portanto, calculando-se as derivadas, igualando-as a zero e resolvendo-se as equações:

$\mathrm{k}^{2} \hat{\rho}-2 \mathrm{bn}(1-\hat{\rho})=0$ e $-\mathrm{k}+\mathrm{n}^{2}=0$, obtêm-se: $\mathrm{n}=\sqrt[3]{\frac{2 b(1-\hat{\rho})}{\hat{\rho}}}, \hat{\rho}>0$ e $\mathrm{k}=\sqrt{\frac{2 b n(1-\hat{\rho})}{\hat{\rho}}}, \hat{\rho}>0$, pois $\mathrm{k}=\mathrm{n}^{2} ; \mathbf{k}$ e $\mathbf{n}$ são, respectivamente, os estimadores do número de plantas úteis da parcela e do número de fileiras de plantio, dessas parcelas, isto é, $\mathrm{f}(\mathrm{n}, \mathrm{k})$ são as coordenadas do ponto mínimo da variância $\mathrm{V}(\hat{m})$, então:

$\mathrm{f}(\mathrm{n}, \mathrm{k})=\mathrm{V}(\hat{m})=\frac{\sigma^{2}}{N}\left(1+\frac{2 b}{n}\right)\left(1+\frac{2 b n}{k}\right)[1+(k-1) \hat{\rho}]$.

Como a área dos experimentos de campo com esses cultivos é função de $\mathrm{N}=\mathrm{rK}$, considerem-se dois experimentos com parcelas de, respectivamente, $\mathbf{k}$ e k' plantas úteis, em $\mathbf{n}$ e n' fileiras, com um total de K e K' plantas por parcela, e N e N' plantas em cada tratamento, isto é: $\mathrm{N}=\mathrm{rK}, \mathrm{N}^{\prime}=\mathrm{r}^{\prime} \mathrm{K}^{\prime}$. Dessa forma, tem-se que:

$$
\frac{N^{\prime}}{N}=\frac{\left(1+\frac{2 b^{\prime}}{n^{\prime}}\right)\left(1+\frac{2 b^{\prime} n^{\prime}}{k^{\prime}}\right)\left[1+\left(k^{\prime}-1\right) \hat{\rho}\right]}{\left(1+\frac{2 b}{n}\right)\left(1+\frac{2 b n}{k}\right)[1+(k-1) \hat{\rho}]}
$$

Como as áreas de cada experimento são proporcionais aos números totais de plantas, tem-se:

$$
\frac{A^{\prime}}{A}=\frac{\left(1+\frac{2 b^{\prime}}{n^{\prime}}\right)\left(1+\frac{2 b^{\prime} n^{\prime}}{k^{\prime}}\right)\left[1+\left(k^{\prime}-1\right) \hat{\rho}\right]}{\left(1+\frac{2 b}{n}\right)\left(1+\frac{2 b n}{k}\right)[1+(k-1) \hat{\rho}]}
$$

Sendo, respectivamente, $\mathbf{r}$ e r' o número de repetições para cada experimento, tem-se que:

$$
\frac{A^{\prime}}{A}=\frac{N^{\prime}}{N}=\frac{r^{\prime} K^{\prime}}{r K} \quad \text { ou seja } \quad \frac{A^{\prime}}{A}=\frac{r^{\prime} K^{\prime}}{r K} \quad \text { ou ainda } \quad \frac{r^{\prime}}{r}=\frac{K}{K^{\prime}} \times \frac{A^{\prime}}{A}
$$

\section{RESULTADOS E DISCUSSÃO}

Como $\mathrm{f}(\mathrm{k})=\mathrm{Y}(\hat{m})=\left(\frac{\sigma^{2}}{N}\right)[1+(\mathrm{k}-1) \hat{\rho}]$, considere-se, por exemplo, $\mathrm{N}=10$ plantas e $\hat{\rho}=+0,050$. É simples verificar que o mínimo da variância se dá para $\mathrm{k}=1$, independentemente do sinal de $\hat{\rho}$. Se, porém, $\mathrm{k}>1$ e $\hat{\rho}>0$, essa variância é função crescente de $\mathrm{k}$. Mas, se $\mathrm{k}>1$ e $\hat{\rho}>0$, ela é função decrescente de k, ou seja:

Tomando $\hat{\rho}=\mathbf{0 , 0 5 0}$

Para $k=1, f(1)=\left(\sigma^{2} / 10\right)$

Para $k=2, f(2)=\left(\sigma^{2} / 10\right) 1,05$

Para $k=5, f(5)=\left(\sigma^{2} / 10\right) 1,20$

Para $\mathrm{k}=10, \mathrm{f}(10)=\left(\sigma^{2} / 10\right) 1,45$

\author{
Tomando $\hat{\rho}=\mathbf{- ~ 0 , 0 5 0}$ \\ Para $\mathrm{k}=1, \mathrm{f}(1)=\left(\sigma^{2} / 10\right)$ \\ Para $k=2, f(2)=\left(\sigma^{2} / 10\right) 0,95$ \\ Para $k=5, f(5)=\left(\sigma^{2} / 10\right) 0,80$ \\ Para $k=10, f(10)=\left(\sigma^{2} / 10\right) 0,55$
}

De modo geral, o mínimo se daria então para $\mathrm{k}=\mathrm{N}$ ou $\mathrm{r}=1$, mas com duas restrições:

a) Deve-se ter $[1+(\mathrm{k}-1) \hat{\rho}] \geq 0$.

b) Deve haver um número considerável de repetições $r \geq \geq 2$, que forneça razoável número de graus de liberdade para o resíduo, pelo menos dez, para que se tenha bons resultados.

Em experimentos de campo com cajueiro comum e anão precoce, Rossetti et al. (1991) e Rossetti et al. (1996) mostraram que o coeficiente de correlação intraclasse $\hat{\rho}$ varia entre 0,0647 e 0,2967 , ou seja: $0,0647<\hat{\rho} \leq 0,2967$.

Tome-se, nesse intervalo, dois experimentos com $\hat{\rho}=0,1243$ e bordadura simples ou completa $(b=1)$ entre as parcelas. No primeiro, cada parcela foi formada por $\mathrm{k}=12$ plantas úteis em $\mathrm{n}=4$ fileiras; no segundo, as parcelas tinham $\mathrm{k}^{\prime}=4$ plantas úteis em n' $=2$ fileiras.

Aplicando a expressão (2), verifica-se que havia um total de $\mathrm{K}_{1}=30$ e $\mathrm{K}_{2}=16$ plantas por parcela, respectivamente, no primeiro e segundo experimentos, ou seja:

$\mathrm{K}_{1}=(4+2)\left(\frac{20}{4}+2\right) \quad \mathrm{K}_{1}=30$, no primeiro, e $\mathrm{K}_{2}=(2+2)\left(\frac{6}{2}+2\right) \mathrm{K}_{2}=16$

no segundo experimento.

Aplicando-se a expressão (3), verifica-se que as variâncias da média de cada tratamento, respectivamente, do primeiro e segundo experimentos, são:

$V_{1}(\hat{m})=\frac{\sigma^{2}}{N} 5,91825 \quad V_{2}(\hat{m})=\frac{\sigma^{2}}{N} 5,4916$

Conclui-se que a variância da média de cada tratamento do primeiro experimento é 7,21\% maior que a do segundo. Logo, havendo diferenças significativas entre os tratamentos de ambos os experimentos, sua detecção é muito mais difícil no primeiro que no segundo.

Aplicando a expressão (4), verifica-se que a relação entre as áreas ocupadas pelos primeiro e segundo experimentos será: $\frac{A_{2}}{A_{1}}=\frac{5,4916}{9,91825}=0,92 \Rightarrow A_{2}=92 \% A_{1}$

Isto é, o segundo experimento ocuparia $92 \%$ da área do primeiro. Como no primeiro haviam $\mathrm{r}=4$ repetições, é possível 
verificar-se, com o auxílio da expressão (5), que o segundo poderia ter:

$$
\begin{gathered}
\frac{r^{\prime}}{r}=\frac{30}{16} \times \frac{5,4916}{5,91825} \Rightarrow \frac{r^{\prime}}{r}=1,7398 \Rightarrow r^{\prime}=1,7398 r \Rightarrow \\
r^{\prime}=1,7398 \times 4 \Rightarrow r^{\prime}=6,9 \Rightarrow r^{\prime} 6 \text { ou } 7 \text { repetições. }
\end{gathered}
$$

Mesmo aumentando o número de repetições, de $\mathrm{r}=4$ para $\mathrm{r}^{\prime}=6$ ou 7 , o segundo experimento ocuparia área $8 \%$ menor do que a do primeiro, com economia de $8 \%$ na área experimental. $\mathrm{O}$ número total de plantas por tratamento será reduzido de $\mathrm{N}=4 \times 30=120$ para $\mathrm{N}^{\prime}=6 \times 16=96$, no caso de $\mathrm{r}=6$, ou $\mathrm{N}^{\prime}=7 \times 16=112$, no caso de $\mathrm{r}=7$ repetições, com redução efetiva de $20,0 \%$ ou $6,7 \%$ no número de plantas, para o novo experimento conforme se usarem $\mathrm{r}=6$ ou 7 repetições. Além disso, o segundo experimento terá maior número de graus de liberdade para estimar o erro experimental, portanto maior precisão na estimativa dos efeitos de tratamentos e na aplicação dos testes estatísticos, facilitando detectar diferenças significativas entre os tratamentos, quando estas existirem.

Tomem-se, agora, dois experimentos com $\hat{\rho}=0,0983 \mathrm{e}$ bordadura simples ou completa $(b=1)$ entre as parcelas. No primeiro, cada parcela foi formada por $\mathrm{k}=20$ plantas úteis em $\mathrm{n}=4$ fileiras; no segundo, as parcelas tinham $\mathrm{k}^{\prime}=6$ plantas úteis em $n '=2$ fileiras.

Aplicando a expressão (2), verifica-se que havia $\mathrm{K}_{1}=42$ e $\mathrm{K}_{2}=20$ plantas por parcela, respectivamente, no primeiro e segundo experimentos, ou seja:

$\mathrm{K}_{1}=(4+2)\left(\frac{20}{4}+2\right) \quad \mathrm{K}_{1}=42$ no primeiro, e $\mathrm{K}_{2}=(2+2)\left(\frac{6}{2}+2\right) \quad \mathrm{K}_{2}=20$, no segundo experimento.

Aplicando a expressão (3), obtêm-se, analogamente ao exemplo anterior, que:

$V_{1}(\hat{m})=\frac{\sigma^{2}}{N} 6,02217 \quad V_{2}(\hat{m})=\frac{\sigma^{2}}{N} 4,97167$

Conclui-se que a variância da média de cada tratamento do primeiro experimento é $17,44 \%$ maior que a do segundo. Isso confirma que parcelas grandes têm maior variância e indica que havendo diferenças significativas entre os tratamentos de ambos os experimentos, sua detecção é muito mais fácil no segundo que no primeiro.

Aplicando a expressão (4), obtém-se: $\frac{A_{2}}{A_{1}}=\frac{4,97167}{6,02217}=$ $0,82=82 \%$, ou seja: $\mathrm{A}_{2}=82 \% \mathrm{~A}_{1}$. Isto é, o segundo experimento ocuparia $82 \%$ da área do primeiro. Como no primeiro havia $\mathrm{r}=5$ repetições, verifica-se, com o auxílio da expressão (5), que o segundo experimento poderia ter:

$\frac{r^{\prime}}{r}=\frac{42}{20} \times \frac{4,97167}{6,02217} \Rightarrow \frac{r^{\prime}}{r}=1,7336 \Rightarrow r^{\prime}=7336 r \Rightarrow r^{\prime}=1,7336 \times 5 \Rightarrow r^{\prime}=8,7 \Rightarrow r^{\prime}=8$ ou 9 repetições

Mesmo aumentando o número de repetições, de r $=5$ para r' $=8$ ou 9 , o segundo experimento teria $82 \%$ da área do primeiro, com economia de $18 \%$ na área experimental. O número total de plantas por tratamento será reduzido de $\mathrm{N}=5 \times 42=210$ para $\mathrm{N}^{\prime}=8 \times 20=160$, no caso de $\mathrm{r}=8$, ou N $\mathrm{N}^{\prime}=9 \times 20=180$, no caso de $\mathrm{r}=9$ repetições, com redução efetiva de $23,8 \%$ ou $14,3 \%$ no número de plantas, para o novo experimento, conforme se usarem $\mathrm{r}=8$ ou 9 repetições. Além disso, o segundo experimento terá maior número de graus de liberdade para estimar o erro experimental, dando maior precisão às estimativas dos efeitos de tratamentos e à aplicação dos testes estatísticos, portanto maior facilidade de detectar diferenças significativas entre os tratamentos, quando estas existirem.

As pesquisas com seringueira, conforme Rossetti \& Pimentel Gomes (1987), são feitas por grande número de experimentos com três a quatro repetições. Analisando-se vários deles, em blocos ao acaso, com $\mathrm{r}=3$ repetições, bordadura simples $(b=1)$ entre as parcelas de $k=16$ plantas úteis em $n=4$ fileiras e $\mathrm{K}=36$ plantas ao todo, cada tratamento era constituído de 108 plantas, detectou-se grande variabilidade dentro desses tratamentos, o que dificulta a detecção de diferenças significativas entre eles. Um experimento nessas condições, com $\hat{\rho}=0,0789$, e parcelas de $\mathrm{k}^{\prime}=4$ plantas úteis em n' $=2$ fileiras, teria $\mathrm{r}^{\prime}=6,8$, podendo-se utilizar $\mathrm{r}=6$ ou 7 repetições. Com $\mathrm{r}=6$ repetições, cada tratamento teria 96 plantas em parcelas de $\mathrm{K}=16$ plantas ao todo, ocupando precisamente a mesma área, porém com as vantagens de ter menor variância dentro das parcelas e de contar com maior número de graus de liberdade para estimar o erro experimental, os efeitos de tratamentos e de outros parâmetros, aumentando, assim, a possibilidade de serem detectadas diferenças significativas entre os efeitos testados. Com r' $=7$ repetições, cada tratamento passaria a ser constituído de 112 plantas, ocupando, portanto, uma área ligeiramente maior (apenas $0,96 \%$ maior que a do experimento original, com três repetições), que, mesmo assim, ainda seria vantajoso, pois o aumento do número de graus de liberdade propiciaria melhor estimativa do erro experimental e dos efeitos de tratamentos, com reflexos positivos na precisão experimental e na qualidade dos resultados obtidos.

Embora se saiba da importância que tem o número de repetições nos experimentos dos diversos ramos da pesquisa, há, às vezes, restrições que impedem o uso do número desejável de repetições, sendo a mais comum relativa à área total do experimento. Assim sendo, a alternativa sugerida neste trabalho, para aumentar o número de repetições e a eficiência dos experimentos, reduzindo, ao mesmo tempo, a quantidade de plantas necessárias e, por vezes, a área experimental, parece razoável, pois permite maior precisão à pesquisa agronômica de campo, com plantas perenes ou frutíferas arbóreas, com custos mais reduzidos.

\section{CONCLUSÕES}

1. Nos experimentos de campo com plantas perenes ou frutíferas arbóreas, a diminuição da variância da média de cada tratamento é obtida pelo uso de parcelas pequenas.

2. Maior número de repetições com parcelas pequenas possibilita diminuir o número de plantas necessárias ao experimento e o tamanho da área experimental, reduzindo os custos da pesquisa. 3. Maior número de repetições com parcelas pequenas permite aumentar a precisão do experimento, das estimativas do erro experimental, dos efeitos de tratamentos e de outros parâmetros, favorecendo detectar diferenças significativas entre os tratamentos, quando estas existem, propiciando maior confiabilidade aos resultados da pesquisa.

\section{REFERÊNCIAS BIBLIOGRÁFICAS}

DAGNELIE, P. Théorie et méthodes statistiques. Paris: Press Agronomiques de Gembloux, 1975. v.2. 
PIMENTEL GOMES, F. O problema do tamanho das parcelas em experimentos com plantas arbóreas. Pesquisa Agropecuária Brasileira, Brasília, v.19, n.12, p.1507-1512, 1984.

PIMENTEL GOMES, F. Curso de estatística experimental. 13. ed. Piracicaba: Nobel, 1990. 468p.

ROSSETTI, A. G. Planejamento de experimentos de nutrição e adubação com plantas perenes arbóreas. Fortaleza: EmbrapaCNPAT, 1994. 50p. (Embrapa-CNPAT. Documentos, 13).

ROSSETTI, A. G.; PIMENTEL GOMES, F. Determinação do tamanho ótimo de parcelas em ensaios agrícolas. Pesquisa Agropecuária Brasileira, Brasília, v.18, n.5, p.477-487, 1983.

ROSSETTI, A. G.; PIMENTEL GOMES, F. A method for the determination of optimum plot size in experiments with rubber tree (Hevea). Journal of Natural Rubber Research, Malaysia, v.2,n.3, p.135-141, 1987.

ROSSETTI, A. G.; ALMEIDA, J. I. L. de; PARENTE, J. I. G.; BARROS, L. de M. Tamanho ótimo de parcela para experimentos com cajueiro comum. Revista Brasileira de Fruticultura, Cruz das Almas, v.13, n.2, p.117-122, 1991.

ROSSETTI, A. G.; BARROS, L. de M.; ALMEIDA. J. I. L. de. Tamanho ótimo de parcelas para experimentos de campo com cajueiro-anão precoce. Pesquisa Agropecuária Brasileira, Brasília, v.31, n.12, p.843-852, 1996. 